\title{
Conversations après un enterrement: l'exquise obscénité de Yasmina Reza et sa «stratégie des poireaux»
}

\section{Montserrat Serrano Mañes}

\section{(2) OpenEdition Journals}

Édition électronique

URL : https://journals.openedition.org/studifrancesi/2964

DOI : 10.4000/studifrancesi.2964

ISSN : 2421-5856

Éditeur

Rosenberg \& Sellier

Édition imprimée

Date de publication : 1 juillet 2013

Pagination : 416-423

ISSN : 0039-2944

\section{Référence électronique}

Montserrat Serrano Mañes, "Conversations après un enterrement: l'exquise obscénité de Yasmina Reza et sa «stratégie des poireaux» », Studi Francesi [En ligne], 170 (LVII | II) | 2013, mis en ligne le 30 novembre 2015, consulté le 31 janvier 2023. URL : http://journals.openedition.org/studifrancesi/2964 ; DOI : https://doi.org/10.4000/studifrancesi.2964

\section{(c)}

Creative Commons - Attribution - Pas d'Utilisation Commerciale - Pas de Modification 4.0 International - CC BY-NC-ND 4.0

https://creativecommons.org/licenses/by-nc-nd/4.0/ 


\title{
Conversations après un enterrement: l'exquise obscénité de Yasmina Reza et sa «stratégie des poireaux»
}

\begin{abstract}
This reflection is built on three fundamental elements: any viewer is a "voyeur", the scene is essentially exhibitionist, the actors' verbal interaction displays and strengthens the intrinsic "obscenity" of the theatrical word. Then comes the setting, - as a premise of reading - of the transgressive and at the same time lenitive specificity of Yasmina Reza's dramatic writing, particularly effective in "Conversations après un enterrement". Proposed prospective bases will allow the analyst to highlight the dramaturgical formalization of the author's neutralizing strategy. All spectators, chosen or accepted, despite the existential possibility of a hyper "witness" indifferent to the contingency of reception, is immersed in the speech and in the flesh of the actors in the original obscenity: see and be seen, a kind of architecture to look at. In addition, the viewer becomes accomplice in initial collusion with the writer and actors, with exchanges, "tête a tête", of beings that boast their affluent talkativeness and unyielding pantomime on the stage. But ultimately, the witty "leeks" strategy will defuse all insubstantial in life.
\end{abstract}

Questa riflessione si articola su tre assi fondamentali: ogni spettatore è un "voyeur", la scena è essenzialmente esibizionista, l'interazione verbale tra gli attori svela e ostenta l'"oscenità" intrinseca alla parola teatrale. Si giunge in seguito alla dimostrazione, come premessa di lettura, della specificità sia trasgressiva che "lenitiva" della scrittura drammatica di Yasmina Reza, particolarmente evidente in Conversations après un enterrement. Le basi previsionali proposte permetteranno all'analista di evidenziare la formalizzazione drammaturgica della strategia neutralizzante dell'autore. Ogni spettatore, scelto o accettato - nonostante la probabilità esistenziale di un iper "testimone" indifferente alle contingenze della recezione -, è immerso nel discorso e nella carne degli attori in un'oscenità originaria: vedere, essere visto, una sorta di architettura dello sguardo. Inoltre, con l'accordo iniziale della scrittrice e degli attori, lo spettatore diviene complice degli scambi, dei tête-à-tête di esseri che esibiscono sulla scena la loro opulenta loquacità e la loro irriducibile pantomima. Tuttavia l'esilarante strategia dei "porri" sarà l'elemento che neutralizza tutte le sconsideratezze della vita.

Je voudrais, pour circonscrire le sens du titre de cet article, indiquer tout d'abord que je pars de l'idée que tout spectateur est un «voyeur», que la scène est exhibitionniste, que l'interaction verbale des acteurs est là pour étaler l'obscénité de la parole; ensuite établir comme prémisse de lecture le caractère transgressif et en même temps «désamorceur» de l'œuvre de Yasmina Reza, ce qui permet à l'analyste de se pencher sur cet aspect concret de sa stratégie théâtrale neutralisante. Nous, spectateurs, nous plongeons avec les paroles et les corps des acteurs, dans une obscénité foncière: regarder, être regardé. Nous assistons, avec la complicité première de l'auteur et des acteurs, à des conversations d'êtres qui déploient sur scène leur incontinence langagière et gestuelle. Mais il existe toujours cet élément déterminant de l'existence quotidienne qui finalement s'impose comme une composante incontournable de l'être humain et de la vie sociale.

Yasmina Reza est une écrivaine dont le rayonnement public et la reconnaissance internationale sont incontestables, et qui cependant est absente - à quelques excep- 
tions près - des livres de texte, des dictionnaires du théâtre, des études dramaturgiques $^{1}$. Une écrivaine dont tout au plus, on trouve quelques lignes par-ci, par-là, qui se penchent - pas trop quand même! - sur Art notamment, sa pièce la plus connue ${ }^{2}$. La question que l'on se pose d'emblée est la suivante: pourquoi cette absence? Son succès apparemment facile gênerait les intellectuels? Son théâtre qui frôle, a-t-on dit, le boulevard, ne gênerait pas assez, comme il est de mise de nos jours? C'est parce qu'elle n'est pas «obscène» au sens propre ou plutôt actuel et réductionniste du terme? Parce qu'elle n'est pas assez difficile? Et la question de Denis Guénoun s'est alors imposée comme une fulguration: «Avez-vous lu Reza?» Question oh combien pertinente si l'on voulait surprendre ou du moins traquer l'obscénité et ses nuances dans cette œuvre limpide et cependant énigmatique.

Le choix de Conversations après un enterrement s'est aussi imposé de manière éclatante: ce titre ne porte-t-il pas en lui-même une sorte d'obscénité préliminaire? D'ailleurs, rien de plus nécessaire que de commencer par le commencement: le fait de choisir sa première pièce théâtrale impliquait en même temps attraper au vol les premiers élans de la voix théâtrale de Reza. Prix Molière de 1987, cette pièce de jeunesse écrite en 1983-1984 promet dans son titre même des non-dits et des rapprochements du tragique et du comique, du grave et du frivole: la mort et la parole en quête de dérision ou d'absolu. Il comporte dans sa dichotomie un germe d'obscénité: parole - dramatique, donc exhibitionniste -, et de surcroît après un enterrement: banalité et futilité des mots qui côtoient la douleur tragique. Enterrement, c'est-à-dire, mort; et qu'est-ce qu'il y a de plus obscène que la mort?

Cependant, le but étant de traquer l'obscénité dans une écriture qui se veut transparente, lumineuse, il fallait vérifier ce que les mots «obscène» et «obscénité» permettent au lecteur/spectateur de dire, et par quel droit chemin - ou plutôt mauvais chemin - peuvent-ils l'engager. Les dictionnaires sont clairs là-dessus: «Obscène: adj. (lat. obscenus, de mauvais augure). Qui blesse ouvertement la pudeur par des représentations d'ordre sexuel: indécent. 2. Par ext.: Qui choque par son caractère scandaleux, immoral. «Cet étalage de richesse est obscène»». Obscénité peut rapprocher le lecteur de son but: «Obscénité: 1 . caractère de ce qui est obscène. 2. Parole, acte obscène». Et Meillet laisse le récepteur accroché à un mince filet de signifiés possibles: «obscenus, a um: T. de la l. augurale de mauvais augure»; par suite dans la langue courante «d'aspect laid ou affreux; qu'on doit éviter ou cacher; obscène». Il ajoute: «la variation obscenus, obscaenus rappelle celle de 'scena', 'scaena', qui semble supposer un intermédiaire étrusque entre le modèle grec et l'emprunt latin». 'Scena', 'scaena', 'scène', (sens propre et figuré) puis «spectacle», «spectateurs». Le mot latin est emprunté au grec $\sigma x \eta \nu \eta$ dans la mesure où ce mot grec a un sens technique et s'applique à des éléments du théâtre.

Comment parler de Yasmina Reza en de tels termes? Or, j'ai essayé de contourner ce que je considérais un problème en m'accrochant au découpage premier: observer, scène, obscénité, laissant ce dernier terme en veilleuse.

Le charme du théâtre de Yasmina Reza reste toujours mystérieux. Du point de vue de la structure, ses pièces présentent une construction qui semble chercher - et atteindre - l'efficacité, en même temps qu'elle renverse les valeurs acquises. Théma-

(1) «...tandis qu’à New-York on la traite avec une grande considération, en France elle ne parvient pas à gagner la faveur de tous, encore moins la sympathie des happy few, des dogmatiques de différents bords ou des esprits chagrins» (CH. BLOTLABARRÈRE, «Yasmina Reza: mesures du Temps», in Nouvelles écrivaines, nouvelles voix?, sous la dir. de Morello, N., et Rodgers, C., Amsterdam-NewYork, Rodopi, 2002, pp. 269-285, p. 272).

(2) Et pourtant son roman Une désolation a remporté un grand succès mondial, mais évidemment, laissant de côté son œuvre aussi bien romanesque que théâtrale, ces dernières années on a appuyé la touche sur le controversé L'aube le soir ou la nuit. 
tique directe, et toutefois insaisissable, style clair, trop évident même, en apparence. Son œuvre - multiple et multiforme - semble appartenir au domaine du théâtre le plus commun, et cependant tout l'en éloigne. Grâce à ce que Guénoun appelle la «stratégie des poireaux» ${ }^{3}$, elle contourne la tragédie, la comédie trop criarde, les moments scabreux.

L'intrigue de Conversations après un enterrement est mince: à la campagne, d'après la didascalie - «Une propriété de famille dans le Loiret», «Pas de réalisme», «Espace ouvert et unique»-, avec une mise en scène toute en suggestions, nous rencontrons six personnages: Nathan, Édith et Alex, frères; Pierre, leur oncle maternel, et sa femme Julienne; et finalement Elisa, ex-maîtresse d'Alex et amoureuse de $\mathrm{Na}$ than. Ils sont là pour l'enterrement du père des frères quadragénaires, qui a voulu être enterré dans le jardin; c'est au moment où l'on est en train de recouvrir de terre le cercueil que nous rencontrons le groupe, et que par la lecture d'une lettre nous entendons pour la première et dernière fois la voix du père que l'on vient d'ensevelir: une lettre adressée à vingt ans au fils à venir, encore imaginaire, Nathan, «mon prodigieux éclat». La pièce commence donc juste 'après' l'enterrement. Et le père sera pour toujours là, sous terre, à leurs côtés, présence continuellement affirmée.

Alex est le frère cadet, un écorché vif, qui se sent mal aimé du père, et qui supporte très mal le fait que Elisa l'ait abandonné et qu'elle lui préfère son frère. Nathan, le bien-aimé, contourne depuis toujours le mal de vivre de son frère, et jusqu'ici il s'est refusé à vivre son histoire d'amour avec Elisa par amour fraternel. Leur soeur Édith, au beau milieu des deux, connaît leurs sentiments et essaie d'éviter les heurts qui pourraient mener à une rupture violente. L'oncle, témoin du passé - rapports du père avec ses enfants, psychologie d'Alex et de Nathan... -, est là pour dire des vérités mais aussi pour apaiser la situation quand elle devient trop conflictuelle. Sa femme, Julienne, est une femme de bon sens, pleine d'ingénuité et de bons sentiments, dont le rôle frôle souvent celui de bouc émissaire. Elisa, le seul personnage qui n'appartient pas à la famille, est l'élément soi-disant perturbateur: arrivée à l'improviste, des problèmes successifs vont empêcher sa sortie du huis clos familial.

Nous voyons vivre et agir ces êtres pendant un jour, nous sentons monter la violence, entraînant un déclenchement brutal, puis nous voyons cette violence se désamorcer et se dissoudre complètement par l'acceptation d'Alex des rapports de Nathan avec Elisa, au lieu d'assister à l'affrontement fratricide auquel on pouvait s'y attendre. Rien de bien grave, donc, et la vie continue.

Il ne serait pas pertinent de s'arrêter sur la structure de la pièce, étant donné que la finalité de cette étude est tout autre. Cependant, je ferai quelques petites remarques qui pourraient expliciter la dynamique des acteurs: ces six personnages, souvent tous rassemblés, parfois réunis en duo, ont comme figure géométrique et scénique de base le triangle, qui les distribue et les case tous: les trois frères, les trois hommes, les trois femmes, le triangle amoureux Alex-Nathan-Elisa. Quant au découpage, ce qui tient lieu de scènes ${ }^{4}$, il est marqué, d'après le texte didascalique, par les jeux de lumière:

(3) C'est-à-dire, selon D. GuÉnoun (Avez-vous lu Reza? Paris, Albin Michel, 2005, p. 50), tous les éléments inclus dans la pièce qui appartiennent à la vie ordinaire et qui ont pour but «l'évitement du tragique».

(4) Conformément aux tendances du théâtre moderne et le nouvel ordre qui respecte les liaisons permanentes entre l'harmonie et le contraste, il n'existe pas un arrangement de scènes continu, mais plutôt juxtaposition. M. Lioure (Lire le théâtre moderne, Paris, Dunod, 1998, p. 132) signale comment «Le procédé du tableau, quelles qu'en soient les ressources et les fonctions techniques, a été pour les dramaturges un moyen privilégié de rompre avec les canons traditionnels de la continuité pour leur substituer une esthétique de la diversité, du changement, de la segmentation, de la brisure et du heurt, mieux accordée au goût et à la sensibilité modernes». 
changements de lieu, changements de décor... L'éclairage ${ }^{5}$ ordonne le mouvement scénique d'un plateau dénué de tout accessoire - ou presque - et dont les différents espaces - campagne, jardin, bois, clairière, terrasse, intérieur de la maison - ne sont qu'insinués, suggérés, voire imaginés.

D'autre part, si ce qui déclenche l'action est l'enterrement du père, la scène sur laquelle pivote la pièce est la rencontre sexuelle de Nathan et Elisa sur sa tombe: rapports sexuels qui vont à l'encontre de toute convenance, et qui au bout du compte modifieront le comportement des personnages.

L'oscillation de Conversations après un enterrement entre le comique et le tragique est ce qui fait sa force. Ou plutôt, c'est de cette oscillation que naît sa puissance. La pente tragique que ces personnages pourraient emprunter n'est qu'insinuée: les rapports avec le quotidien aussi bien que la gestuelle des personnages désamorcent à chaque pas ce que le langage souvent brutal est sur le point de déchaîner.

En reprenant le fil de l'invocation manifestée dans le titre, je vais essayer de me raccrocher à deux des éléments que je viens de citer. Tout d'abord, et puisque le théâtre est parole, dite pour être écoutée par nous, «obscènes» voyeurs invités, je cernerai ce que de violent, de brutal ou d'obscène transmet le discours des personnages.

Un seul personnage semble accaparer, pendant la plupart du temps, le langage violent et brusque et les inconvenances langagières: le discours d'Alex véhicule son mal de vivre et son ressentiment. Nous pouvons considérer, avec Ryngaert ${ }^{6}$, que «La parole d'un personnage organise son rapport au monde dans l'usage qu'il fait du langage».

Dans ses premiers échanges verbaux avec sa sœur Édith, il emploie un registre de langue familier et populaire, voire vulgaire, par lequel il exprime son malaise envers un personnage absent, duquel nous ne savons rien si ce n'est par ces mots mêmes; l'effet de répétition ajoute à la scène un surplus de violence difficilement contenue:

ALEX. Elle est partie?

EDITH. Non.

ALEX. Dis-lui de foutre le camp.

EDITH. Arrête...

ALEX. Dis-lui de foutre le camp. S'il te plait.

Silence

EDITH. Voulez-vous que je fasse du café?

ALEX. Il y a là-dedans une indécence! (CE: 8)

Pour la première fois, la «stratégie des poireaux» fait surface: la sœur est là pour tenter de désamorcer la crise qui s'annonce, en proposant quelque chose de banal, un petit café. Banalité qui permettra un postérieur tête à tête entre Nathan et Elisa, conjoncture qui permettra à la jeune femme de lui avouer son amour: «Durant ces années, je n'ai pensé qu'à une seule chose, te revoir, je n'ai eu qu'une obsession, te revoir, te voir, entendre ta voix... J'ai vécu hantée par toi, incapable d'aimer qui que ce soit...» (CE: 10). La séquence du café se poursuit entre les trois frères, tout en

(5) P. Pavis (L'Analyse des spectacles, Paris, Nathan, 1996, pp. 178-179) indique comment l'éclairage favorise l'inscription de la dramaturgie dans le temps. Comme il le signale pertinemment, «La lumière facilite la compréhension. Si l'objet éclairé est bien contrasté, il sera clairement reconnu. La lumière est responsable du confort ou de l'inconfort de l'écoute, de la compréhension plus ou moins rationnelle d'un événement».

(6) J.-P. RYNGAERT, Introduction à l'analyse du Théâtre, Paris, Bordas, 1991, p. 90.

(7) Nos citations renvoient à l'édition Conversations après un enterrement, Actes Sud (papiers), Paris, 1986. Dorénavant, le titre de la pièce sera indiqué par le sigle CE. 
conservant le registre de langue, dans lequel la susceptibilité d'Alex ne cesse pas de se manifester:

Je le prends pas mal, qu'est-ce que j'ai dit? Simplement tu as l'air de considérer comme une révélation que je sache faire du café, n'importe quel con peut faire du café, c'est quand même pas un tour de force... Déjà dans la cuisine, tu m’as demandé si je savais! (CE: 10-11)

La «stratégie des poireaux» se déploie dans toute son ampleur du début à la fin de la scène 6, en se prolongeant dans ses effets tout au long de la scène 8: Nathan arrive chargé de légumes pour préparer un pot-au-feu; tout le monde se met à les éplucher, ce qui fait que la conversation tourne de manière naturelle sur la platitude prosaïque de ce qu'ils font, enlevant sa violence à toute phrase ou intervention qui pourrait déclencher des séquelles proches du drame. Alex continue avec ce que son oncle appelle ses «louanges de serpent à sonnettes» (CE: 36) adressées à son frère Nathan, et à manifester par un langage cru et désobligeant son malaise profond, celui que lui cause la liaison de son frère avec Elisa:

ALEX. Faites ce que vous voulez, je m'en fous totalement. matin?

EDITH. On peut les laisser à Vacher, il sait où est sa voiture. Il n'est pas fermé le lundi

ALEX. Je n'en sais rien. Je m'en fous.

EDITH. Merci pour ton aide...

Elle repart.

ALEX. Elle peut bien se démerder toute seule, non?!... Elle nous fait chier avec sa bagnole pourrie!!! (CE: 35)

Car Alex a tout deviné: il sait que Nathan et Elisa sont en train de faire l'amour, et il le dit brutalement à Julienne. «Au théâtre, la parole est action»", et en fait, on perçoit l'effet perlocutoire ${ }^{10}$ de cet échange verbal comme une agression provocante et gratuite d'Alex envers Julienne. En focalisant sa rage sur sa tante, Alex réalise un acte de violence dans lequel la parole se rapproche jusqu'à presque se confondre, avec l'action, avec le geste:

JULIENNE. Où est Elisa?

ALEX. Elle se tape mon frère.

JULIENNE. Pardon?!

ALEX. Elle est en train de se taper mon frère.

JULIENNE. Je ne comprends pas!

ALEX. Mais si, Julienne, vous avez très bien compris. Vous n'êtes pas sourde?

JULIENNE. Mais où?!

ALEX. Ah! Ah! Ah!... J'adore cette question! Ah! Ah!... Vous êtes très rapide, quand même! (CE: 34)

(8) P. Pruner, L'analyse du texte de théâtre, Paris, Dunod, 1998, p. 106.

(9) D'après A. UBERSFELd (Lire le théâtre III, Paris, Belin, 1996, p. 100), «Le perlocutoire, c'est la production d'un effet, effet réel sur le spectateur, effet simulé sur le partenaire, avec cette restriction d'importance que les effets ne sont pas les mêmes». Le discours d'un personnage peut angoisser de manière fictive son partenaire sur scène, mais l'émotion qu'il produit sur le spectateur n'est jamais l'an- goisse ou la peur.

(10) Sur l'utilisation de la gestuelle et les jeux de physionomie, vid. P. LARTHOMAs, Le Langage dramatique, 1980 (1972), Paris, PUF, pp. 81-106, où l'auteur fait remarquer leur importance dans l'interlocution: «L'attention du spectateur est trop vive et l'acteur trop en vedette pour que le moindre de ses gestes n'ait pas tendance à prendre une valeur particulière» (Ibid., p. 88). 
Le fait de prendre sa tante Julienne comme bouc émissaire aura ainsi deux effets: d'un côté celui de faire monter d'un cran la violence ambiante, d'un autre celui de faire baisser la pression, la réaction postérieure de Julienne en pleurs ayant un effet de soupape: au moment où la situation semble prête à éclater, tout se calme à nouveau. La dédramatisation opérée par Reza repose ici sur une déviation du tragique qui survole la scène: à l'accroissement de la tension entre les deux frères répond l'explosion d'un troisième personnage latéral, extérieur au conflit. L'appui sur des éléments de la vie ordinaire - faire du café, préparer une tisane, éplucher des légumes, parler de jeux de table... - renvoie en même temps à la stratégie mise en place par l'auteur pour estomper le tragique:

JULIENNE. ....Le petit commence à peine à parler. Il dit «papa», il dit... Ce qui ne signifie pas grand-chose d'ailleurs, car les enfants les plus... enfin je veux dire les enfants qui tardent sont souvent plus volubiles, en fin de compte!

ALEX. Vous avez dû parler assez tard, alors?

[...]

EDITH. Bon, ça suffit maintenant...

ALEX. Quoi «ça suffit!»? Qu'est-ce qu'il y a? Vous me faites chier!

[...]

EDITH. Va nous faire un café, puisque tu sais si bien le faire...

NATHAN. Une tisane plutôt! (CE: 46-47)

Édith, la sœur, remplit aussi une fonction de dérivation du pathétique. Ses interventions dialogales cherchent à avertir les frères de leur inconduite, mais à chaque fois le résultat est celui de l'évacuation du tragique, soit par le glissement du dialogue vers le comique ou par l'usage langagier de la brutalité. Ainsi, quand elle veut que Nathan soit conscient du moment critique qui approche, son frère Alex sachant qu'il a fait l'amour avec Elisa, elle lui raconte l'aventure de leur père avec sa pédicure Madame Natti: une nouvelle anti-tragique, dont les composantes comiques - nom et profession de la femme, souvenir du père pris en «flagrant délit» et habillé de manière incongrue... - font disparaître toute possible 'pathétisation', faisant dériver le conflit vers l'inconsistance. Du point de vue de la langue actualisée, Alex est certainement celui qui accumule la plupart de la violence verbale de la pièce, mais Édith et Pierre font aussi usage de cette violence discursive, ce qui a comme conséquence encore une fois le déplacement de la tension et l'évitement de l'affrontement entre les frères:

PIERRE. Je suis fatigué mon vieux, tu sais. J'ai plus la force de jouer au con. moi?

ALEX. Tu me dis que j'ai quelque chose à dire. Je te demande quoi? Si tu sais mieux que

EDITH. Si tu n'as rien à dire, ferme-la! Je ne vois pas pourquoi tu nous emmerdes!

ALEX. Et vlan!... Je ne te connaissais pas, ma douce Edith, ce langage... (CE: 53)

Et c'est encore par les mots d'Édith cumulant toute l'agressivité et la brutalité qui animent les rapports entre Nathan et Alex, que le moment majeur est évidé de toute possibilité de dérapage vers la violence physique: Édith assume par ses gestes et par ses paroles l'écart du rituel social et réfrène le mécanisme dramatique:

NATHAN. Si Elisa reste, elle n'ira pas dans cette chambre.

EDITH. Elle ira où, alors?!

NATHAN. Elle ira dans la mienne.

EDITH. Et toi?

NATHAN. Dans la mienne aussi, où veux-tu que j'aille? En d'autres termes, nous passerons la nuit ensemble... Si Elisa reste! 
Silence.

EDITH. J'ai l'impression de rêver... (À Elisa) qu'est-ce que tu fais?! Dis quelque chose! Silence

EDITH. Mais dis quelque chose! Tout le monde décide pour toi, et toi tu es là comme un marbre! Parle!

NATHAN. Je ne vois pas pourquoi tu te mets dans cet état...

EDITH. Je ne comprends plus rien! J'ai l'impression de vivre dans un monde de fous!... Le jour de l'enterrement de papa! (Elle pleure.)

[...]

EDITH. Il faut que tu couches avec cette putain?!... Mais dis quelque chose Elisa! Je t'en supplie, dis quelque chose!... Papa... Papa, viens!... J'ai envie de mourir... (CE: 54)

Parallèlement à la mise en acte du langage, les faits et la gestuelle des personnages traduisent aussi ce que d'obscène ou de hors la norme véhicule la pièce. Ainsi, la scène 5, occupée par le triangle féminin, Julienne-Édith-Elisa, pivote entre la banalité du quotidien et la sexualité: thème inconvenant sans doute un tel jour, mais qui permet de saisir les personnalités et les états d'âme des trois femmes. Du point de vue dramaturgique, la présence de Julienne, la tante, s'avère fondamentale: elle rattache la pièce au jeu dramatique classique, mais aussi, à travers son jeu gestuel, au théâtre de boulevard. Cette présence gênée, qui dit l'inconvenance de la conversation, déclenche en même temps le sourire: double incorrection, qui compromet le spectateur lui-même. Les légers quiproquos dans lesquels tombe Julienne, la description de ses liaisons amoureuses - «Elisa. Trois maris. Vous avez travaillé pour nous, si j'ose dire! Julienne. Le jour de mes dix-huit ans, une voyante extra-lucide m'a prédit le couvent. Je n'étais pas une beauté mais tout de même! Alors je me suis lancée, en quelque sorte, dans une contre-offensive...» (CE: 22) -, ses interruptions discursives provoquées par la gêne de ce qu'elle entend ${ }^{11}$ (CE: 23-24), l'installent sur scène en ce qu'elle-même nomme «position d'auditeur involontaire» (CE: 26), ce que Reza met en relief dans les didascalies, et au bout du compte en «commodité» dramatique, permettant ainsi de faire part aux spectateurs de la trame amoureuse de la pièce:

EDITH. (à Julienne, qui en dépit de sa gêne et d'une croissante incomprébension, s'efforce d'avoir l'air neutre). Cette femme, ma chère Julienne, a rendu fous mes deux frères.

[...]

ELISA. Tu as tort. J'aimerais que tu aies raison, mais tu as tort... (À Julienne) Si vous le permettez madame, je vais rétablir pour vous: j'ai simplement vécu avec Alex, et je suis moimême «éperdue d'amour» pour Nathan. Vous avouerez que c'est assez différent.

Julienne sourit poliment. (CE: 25)

Mais du point de vue des faits et des gestes qui par leur profonde inconvenance se rattachent à l'obscénité, à l'indécence, c'est la scène sexuelle entre Elisa et Nathan qui tient le haut du pavé. La vie et la mort se retrouvent et se confondent sur la tombe du père, le passé et l'avenir se superposent. Aux pensées macabres d'Alex -«Je ne peux plus regarder ce bois sans imaginer papa en train d'étouffer dessous...C'est une folie de l'avoir enterré ici...Vous ne ressentez pas cette oppression vous? Je vois sa tête, les narines pleines de terre, avec le bruit sourd des oiseaux...» (CE: 11) - s'ajoute l'image vivante du couple en train de faire l'amour sur la tombe:

(11) En prenant comme exemple L'École des femmes de Molière, Guénoun s'appuie sur Bakhtine pour théoriser sur l'inclusion dans l'invention dramatique du bas corporel, ressort de base du comique. Et, dans Conversations après un enterrement, comme il le dit à propos de la pièce moliéresque, «Il y a du bas là-dedans» (D. GuÉNOUN, op. cit., p. 216). 
NATHAN. Non. Je n'ai pas envie que tu me laisses... (Un temps.) Tu es plus interdite que jamais, Elisa. Mais aujourd'hui je n'ai pas envie que tu me laisses... (Un temps) Tu sais à quoi je pense? Un acte aussi contraire à la raison...Que j'ai envie de te prendre. Là. Sur sa tombe... Chasser une douleur par une autre.

Elle s'approche tout près de lui.

ELISA. Je veux bien être ta douleur, Nathan...

Il l'embrasse avec passion, et commence à la déshabiller.

Noir.

Exquise obscénité qui fait le noir sur cette scène torride!

L'obscénité de la mort, côtoyant tout au long de la pièce les petits riens de la vie quotidienne, trouve ici son acmé, et souligne l'écart de tout rituel social en rattachant la scène non au bas corporel ${ }^{12}$ comme ressort du comique, mais comme élément du tragique. D'autres interventions gestuelles tendent plutôt vers le comique, soulignant ainsi à tout moment la coexistence de deux réalités, celle de la mort et celle de la vie. L'oncle et la tante sur la route esquissant une scène cocasse, par exemple:

JULIENNE. ...j'ai quelque chose au cœur j'en suis sûre. Tiens, touche...Pas comme ça!... (Petit rire) Pierre, écoute, sur la route!

PIERRE (qui passe sa main sous le chemisier). Mais combien d'épaisseurs tu as?! (CE: 12)

Conversations après un enterrement est un exemple de ce qui constitue l'une des caractéristiques du théâtre de Reza: sous des allures d'élégance normative, coexistent le sourire et le sérieux; on frôle toujours la tragédie et elle est toujours écartée, la «stratégie des poireaux» jouant en faveur du désamorçage des conflits. Les personnages, s'exprimant dans l'entre-deux du temps qui s'écoule, avec une grande liberté de ton et utilisant un langage familier, soulignent les écarts de la norme sociale, sans trop pencher vers la cruauté ni l'obscénité excessivement voyante. Comme le dit Ch. Blot-Labarrère en parlant de Hammerklavier, «les héros et les hérö̈nes font leur miel de l'impertinence, de l'irrespect, de l'ironie, de la maxime piquante, de leurs erreurs, du mot d'esprit où Freud voyait une affirmation du principe de plaisir». Voilà le défi transgressif de Reza qui, en faisant l'éloge subreptice de la futilité, refuse de tomber dans le sérieux absolu et étouffe savamment violence, brutalité, et obscénité. L'élégance et la liberté dans le traitement du thème du deuil mettent en évidence et sa sensibilité et ses facultés dramaturgiques pour engager magistralement sur scène des êtres vivants et crédibles. 